Pesq. Vet. Bras. 37(1):83-90, janeiro 2017 DOI: $10.1590 / \mathrm{S} 0100-736 \mathrm{X} 2017000100014$

\title{
Biometria corporal e parâmetros hematológicos de Trachemys scripta elegans e Trachemys dorbignyi (Testudines: Emydidae) criadas em cativeiro em Petrolina, Pernambuco ${ }^{1}$
}

\author{
Adriana Gradela ${ }^{2 *}$, Viviane N. Souza ${ }^{3}$, Manueli M. de Queiroz ${ }^{2}$; Aline da C. \\ Constantino $^{2}$, Claudio G.C. Bandeira ${ }^{2}$, Marcelo D. de Faria², Liliane Milanelo ${ }^{4}$, \\ Vanessa S. Franzo ${ }^{5}$ e Fábio Mathias Corrêa ${ }^{6}$
}

\begin{abstract}
Gradela A., Souza V.N., Queiroz M.M., Constantino A.C., Bandeira C.G.C., Faria M.D., Milanelo L., Franzo V.S. \& Corrêa F.M. 2017. [Body biometry and hematological parameters of Trachemys scripta elegans and Trachemys dorbignyi (Testudines: Emydidae) reared in captivity in Petrolina/PE, Brazil.] Biometria corporal e parâmetros hematológicos de Trachemys scripta elegans e Trachemys dorbignyi (Testudines: Emydidae) criadas em cativeiro em Petrolina, Pernambuco. Pesquisa Veterinária Brasileira 37(1):83-90. Laboratório de Anatomia dos Animais Domésticos e Silvestres, Colegiado de Medicina Veterinária, Fundação Universidade Federal do Vale do São Francisco, Rodovia $407 \mathrm{Km} \mathrm{12}$, Lote 543, Projeto Nilo Coelho C1, Petrolina, PE 56300-000, Brazil. E-mail: agradela@hotmail.com

This study aimed to evaluate the body biometry and hematological profile of Trachemys scripta elegans $(\mathrm{N}=28)$ and Trachemys dorbignyi $(\mathrm{N}=22)$ reared in captivity in the Brazilian submedium northeastern semi-arid region in the Valley of the São Francisco river. It aimed to establish basic health blood values and generate useful data on the comparative physiology of Testudines. After 120-day adaptation and 24-hour fasting, $2.5 \mathrm{~mL}$ of blood were collected from the dorsal occipital sinus and deposited into a tube with sodium heparin for evaluation, following, of hematological levels. The red blood cell count (RBC) and GLC was conducted in a Neubauer chamber, the hemoglobin level (HGB) was supplied by the cyanmethemoglobin method and the hematocrit (HCT) was obtained by the microhematocrit technique. Based on the RBC, the hematimetric were mathematically established. Body biometry were also evaluated: a) body mass (BM, g); b) maximum dimensions of the carapace [length (MLC, $\mathrm{cm}$ ) and width (MWC, $\mathrm{cm}$ )]; c) maximum dimensions of plastron [length (MLP, $\mathrm{cm}$ ) and width (MWP, $\mathrm{cm}$ )]; d) total length of tail (TLT, $\mathrm{cm}$ ); e) linear length from the base of the tail to the cloacal orifice ( $\mathrm{LPrC}, \mathrm{cm})$; f) linear length from the cloacal orifice to the extremity of the tail $(\mathrm{LPoC}, \mathrm{cm})$. T. scripta elegans showed higher values $(\mathrm{P}<0.05)$ for biometrics, while TLT and LPrC were higher $(\mathrm{P}<0.05)$ in T. dorbignyi. The hematological values did not differ $(\mathrm{P}>0.05)$ among species. The results show that most of the variation found between T. scripta elegans and T. dorbignyi is explained by the biometric variables and that some hematologic correlations characterize interspecies differences. It was conclude that the results shed light on benchmarks for these species kept in captivity in the northeastern semi-arid region and serve as a model for intra and interspecies comparative physiology.
\end{abstract}

INDEX TERMS: Trachemys scripta elegans, Trachemys dorbignyi, Testudines, Emydidae, body biometry, hematology, red cell indices, reptiles.

\footnotetext{
${ }^{1}$ Recebido em 1 de fevereiro de 2016.

Aceito para publicação em 9 de agosto de 2016.

${ }^{2}$ Colegiado de Medicina Veterinária, Universidade Federal do Vale do São Francisco (Univasf), Rodovia $407 \mathrm{Km} \mathrm{12,} \mathrm{Lote} \mathrm{543,} \mathrm{Projeto} \mathrm{Nilo} \mathrm{Co-}$ elho C1, Petrolina, PE 56300-000, Brasil. *Autor para correspondência: agradela@hotmail.com

${ }^{3}$ ALPHA Laboratório de Análises Clínicas Veterinário, Av. Barão do Rio Branco 5, Centro, Petrolina, PE 56304-310, Brasil.
}

\footnotetext{
${ }^{4}$ Centro de Recuperação de Animais Selvagens, Parque Ecológico do Tietê, Departamento de Águas e Energia Elétrica (CRAS-PET/DAEE), Rua Guira Acangatara 70, Engenheiro Goulart, Guarulhos, SP 03719-000, Brasil.

${ }^{5}$ Universidade Federal de Mato Grosso, Rua Fernando Corrêa da Costa 2367, Bairro Boa Esperança, Cuiabá, MG 78060-900, Brasil.

${ }^{6}$ Universidade Estadual de Santa Cruz, Rodovia Ilhéus-Itabuna, Bairro Salobrinho, Ilhéus, BA 45665-900, Brasil.
} 
RESUMO.- Este estudo objetivou avaliar a biometria corporal e o perfil hematológico de Trachemys scripta elegans $(\mathrm{N}=28)$ e de Trachemys dorbignyi $(\mathrm{N}=22)$ criadas em cativeiro na região do submédio do Vale do São Francisco, semiárido nordestino brasileiro, visando estabelecer valores sanguíneos básicos de saúde e gerar dados úteis na fisiologia comparativa de Testudines. Após 120 dias de adaptação e jejum de 24 horas, 2,5 mL de sangue foram coletados do seio occipital dorsal e depositados em tubo com heparina sódica para a avaliação, na sequência, dos níveis hematologicos. A contagem total de eritrócitos (CTE) e global de leucócitos (CGL) foi realizada em câmara de Neubauer; a dosagem de hemoglobina (HGB) pelo método da método da cianometahemoglobina e o hematócrito (HCT) através da técnica do microhematócrito. A partir da CTE estabeleceram-se matematicamente os índices hematimétricos. A biometria corporal também foi avaliada: a) massa corporal (MC, g); b) dimensões máximas da carapaça [comprimento (CMC, $\mathrm{cm}$ ) e largura (LMC, $\mathrm{cm})]$;c) dimensões máximas do plastrão [comprimento (CMP, cm) e largura (LMP, cm)]; d) comprimento total da cauda (CTC, $\mathrm{cm}$ ); e) comprimento linear da base da cauda ao orifício cloacal $(\mathrm{CprC}, \mathrm{cm})$; f) comprimento linear do orifício cloacal a extremidade da cauda(CpoC, $\mathrm{cm}$ ). T. scripta elegans apresentaram valores maiores $(\mathrm{P}<0,05)$ para a biometria corporal, enquanto que o CTC e CprC foram maiores $(\mathrm{P}<0,05)$ em $T$ dorbignyi. Os níveis hematológicos não diferiram $(\mathrm{P}>0,05)$ entre as espécies. Os resultados demostram que a maior parte da variação observada entre T. scripta elegans e T. dorbignyi é explicada pelas variáveis biométricas e que algumas correlações hematológicas caracterizam diferenças interespecíficas. Conclui-se que os resultados lançam luz sobre valores de referência para estas espécies mantidas em cativeiro na região do semiárido e servem como um modelo para a fisiologia comparativa intra e interespécies.

TERMOS DE INDEXAÇ̃̃O: Trachemys scripta elegans, Trachemys dorbignyi, Testudines, Emydidae, biometria corporal, hematologia, índices eritrocitários, répteis.

\section{INTRODUÇÃO}

0 gênero Trachemys, atualmente com 15 espécies, é representado no Brasil apenas por Trachemys adiutrix, restrita ao estado do Maranhão e Piauí (Batistella et al. 2008), e por Trachemys dorbigni, popularmente conhecida como "tigre d'água" e distribuída no Rio Grande do Sul, norte da Argentina e Uruguai (Fritz \& Havas 2007). Outra espécie do gênero, T. scripta elegans, também conhecida como tartaruga-de-orelha-vermelha, embora seja uma espécie exótica e invasora no Brasil, pois é originária do sul dos Estados Unidos da América e do norte do México, se tornou a mais comercializada como pet devido ao tráfico internacional de animais (Alves 2013). Sua excelente adaptação e amplitude alimentar representam riscos a espécies nativas como T. dorbignyi, por competição interespecífica ou pela extinção genética por hibridização (Fonseca 2001, Primack \& Rodrigues 2001). 0 contato entre ambas ocorre quando, ao atingir o tamanho adulto a T. scripta elegans deixa de ser atraente como animal de estimação e é abandonada em lagoas, lagos, rios e corpos d'água. Embora não seja considerada uma espécie ameaçada de extinção, T. dorbignyi tem sofrido impactos negativos causados pela ação antrópica (Silveira et al. 2012).

Conhecer a saúde dos animais é de vital importância para a manutenção de animais saudáveis como pet ou em cativeiro; monitoramento de animais doentes (Pires et al. 2006), avaliação da resposta a tratamentos; realização de diagnósticos ou prevenção de doenças (Metin et al. 2006). Em tartarugas o estado de saúde pode ser avaliado através do hemograma (Pires et al. 2006, Deem et al. 2009), por isto os valores hematológicos têm sido investigados em tartarugas marinhas (Pires 2007, Casal et al. 2009, Deem et al. 2009, Pires et al. 2009, Santos et al. 2009, Snoddy et al. 2009, Flint et al. 2010) e de água doce (Brites 2002, Tesserolli 2004, Hidalgo-Vila et al. 2007, Ferronato 2008, Silva 2011). Contudo, no gênero Trachemys os poucos estudos existentes limitam a discussão dos resultados (Alves 2013), pois fatores como alterações no habitat, introdução de espécies invasoras, poluição ambiental, uso insustentável, mudança climática global (Gibbons et al. 2000, Ruiz et al. 2002), sexo, tamanho, idade e alimentação (Deem et al. 2009); técnicas utilizadas, tempo da amostra, manejo do animal, esforço físico, estado nutricional, condição fisiológica e mental, sazonalidade e contenção química podem levar a grandes variações nos testes realizados por diferentes laboratórios (Hawkey \& Dennet 1989). Por isto, embora os estudos hematológicos comparativos de animais doentes e sadios possam gerar informações importantes para o manejo e conservação das espécies, estas informações terão aplicabilidade limitada se não for estabelecido o padrão de normalidade para determinada população, bem como a fisiologia e as alterações patológicas e como estas se refletem nos parâmetros laboratoriais (Santos et al. 2009).

Apesar de muitos dos métodos empregados em mamíferos poderem ser aplicados em répteis (Falce 2000), na herpetologia a patologia clínica ainda é uma área insipiente (Divers et al. 1996, Cubas \& Baptistotte 2006) e de bibliografia restrita (Campbell 2004). Sabe-se que cuidados devem ser tomados com o local de coleta sanguínea (Medeiros et al. 2012); escolha do anticoagulante (Campbell 2004, Padilla et al. 2009) e necessidade de adaptação da contagem global de células em câmaras de Neubauer (Almosny \& Monteiro 2007). Soma-se a isto a carência de informações sobre a situação atual da interação populacional entre $T$. scripta elegans e T. dorbignyi, para que se possa realizar a implantação de práticas de manejo e conservação de ambas na natureza ou em cativeiro.

Por isto, este estudo objetivou avaliar os valores hematológicos de tartarugas T. scripta elegans e T. dorbignyi criadas em cativeiro em Petrolina (PE) visando contribuir com o estabelecimento de parâmetros de referência e, assim, disponibilizar respaldo científico para a carência de informações e contribuir com estudos comparativos interespecíficos.

\section{MATERIAL E MÉTODOS}

Amostras. Vinte e oito tartarugas da espécie Trachemys scripta elegans e vinte e duas Trachemys dorbignyi recebidas pelo Centro de Triagem de Animais Silvestres (CETAS) do Parque Ecoló- 
gico do Tietê, Guarulhos/SP (2329'23.15”S e 46³1'10.90”W) (Licença No. 131/2013) foram transportadas sob autorização prévia do IBAMA (Processo SMA/DeFau no 13461/2012) até o Campus de Ciências Agrárias da Universidade Federal do Vale do São Francisco, Petrolina/PE (9॰23'34"S e $\left.40^{\circ} 30^{\prime} 28^{\prime \prime} \mathrm{W}\right)$ e criadas em cativeiro. 0 transporte foi realizado em caminhão baú, dentro do qual os espécimes foram contidos em gaiolas que permitiam ampla movimentação com baixa densidade de carga, boa ventilação, além de permitir o fornecimento de alimentação. A cada 6 horas de viagem os animais eram molhados e recebiam alimentação de origem vegetal - manobras importantes para reduzir o estresse, injúrias e até o óbito. Assim, vale salientar que todos os indivíduos foram recepcionados íntegros e livres de mutilações. A viagem teve duração de 48 horas, desde o Parque Ecológico do Tietê, em Guarulhos/SP, até o Campus de Ciências Agrárias da Univasf, Petrolina/PE. À recepção, em Petrolina, os animais foram descarregados com o mínimo de barulho e movimentação e foram acomodados em ambiente mais confortável composto por um aquaterrário, com profundidade mínima da água de $25 \mathrm{~cm}$, acesso a luz solar direta e alimentação industrializada comercial fornecida uma vez ao dia. A manutenção, com substituição total da água e limpeza do recinto era promovida a cada 48 horas. Para evitar proliferação de microrganismos, a água e o recinto eram periodicamente tratados com solução aquosa de azul de metileno. Este estudo foi aprovado pelo SISBIO/IBAMA (protocolo nํ 38601-1) e pelo Comitê de Ética e Deontologia em Estudos e Pesquisas (CEDEP) da Univasf (protocolo no 0001/130314).

Análises biométricas. Após 120 dias de adaptação ao cativeiro, animais clinicamente saudáveis (considerando-se a condição corporal; ausência de ectoparasitas, tumores e lesões cutâneas), com o auxílio de uma fita métrica e paquímetro de aproximação milimetral, tiveram determinadas a biometria corporal com aferições das dimensões máximas da carapaça [comprimento (CMC, $\mathrm{cm}$ ) e largura (LMC, cm)]; do plastrão [comprimento (CMP, cm) e largura (LMP, cm)] (Malvasio et al. 1999) e a biometria da cauda [comprimento total (CTC, cm)]; comprimento linear da base ao orifício cloacal (CprC, cm); e o comprimento linear do orifício cloacal a extremidade da cauda $(\mathrm{CpoC}, \mathrm{cm})]$. A massa corporal (MC, gr) foi avaliada diretamente no visor da balança de precisão analítica (Bioprecisa ${ }^{\circledR}$, Labmais Ltda., Curitiba/PR, Brasil ).

Análises laboratoriais. Os animais foram mantidos por 24 horas de jejum e, então, trazidos ao Laboratório de Anatomia de Animais Domésticos e Silvestres da Universidade Federal do Vale do São Francisco para realização da coleta de sangue à temperatura local de $24^{\circ} \mathrm{C}$. Após serem seguros com ambas as mãos em torno da carapaça e do plastrão nos dois lados do corpo, cranialmente as patas traseiras, os espécimes tiveram a cabeça gentilmente tracionada no sentido cranial, utilizando-se um pequeno seguimento de esparadrapo para que o pescoço ficasse completamente estendido e o seio venoso supraoccipital bilateralmente preenchido de sangue. A agulha foi inserida, perpendicularmente na superfície dorsal do pescoço, a 1,0 cm a partir da linha média cervical dorsal de cada lado do ponto médio da linha. Em tartarugas menores esta distância foi de $0,5 \mathrm{~cm}$ da linha média cervical (Owens \& Ruiz 1980). Aproximadamente 2,5mL de sangue foram coletados utilizando-se seringas de 5,0mL e agulhas (25x7) descartáveis. Imediatamente após, o sangue foi depositado em tubo contendo heparina sódica para a realização, na sequência, do hemograma. As análises foram processadas no Laboratório de Análises Clínicas Veterinário - ALPHA, Petrolina/PE.

A concentração de hemoglobina (HGB, g/dL) foi determinada pelo método da cianometahemoglobina (Campbell 1996) utilizando-se o kit comercial (Labtest ${ }^{\circledR}$, Lagoa Santa/MG). Em tubos de ensaio identificados, $5 \mathrm{~mL}$ do reagente de cor da hemoglobina foram adicionados a $20 \mu \mathrm{L}$ da amostra. Na sequência os tubos foram homo- geneizados e centrifugados e o sobrenadante submetido à leitura em espectrofotômetro (Espectrofotômetro Digital Faixa 325-1000NM - 722 $\mathrm{G}^{\circledR}, \mathrm{E}$-Labcommerce) a um comprimento de onda de 540nm.

As contagens totais de eritrócitos (CTE) e de leucócitos (CGL) foram realizadas de modo manual utilizando-se câmera de Neubauer. Com uma pipeta automática $20 \mu \mathrm{L}$ da amostra foram misturados a $4 \mathrm{~mL}$ de diluente isotônico de Natt \& Herrick (1952) em um tubo de ensaio. Após homogeneização da solução, preencheuse a câmara de Neubauer e os eritrócitos e os leucócitos foram contados por milímetro cúbico, segundo a técnica de Almosny \& Monteiro (2007). Desta forma, em cinco pequenos quadrantes do quadrante central da câmara contou-se os eritrócitos e, considerando-se a diluição 1:100, aplicou-se a fórmula CTE $\left(\times 10^{5} / \mu \mathrm{L}\right)=$ no eritrócitos contados $\times 100 \times 10 \times 5$ (onde 100 é a diluição, 10 a altura da câmara e 5 a área contada). Por outro lado, para a CGL $\left(\mathrm{x} 10^{3} / \mu \mathrm{L}\right)$ foram contadas todas as células contidas em todos os 25 quadrados multiplicando-se o total pelo fator 1.000 .

A partir da determinação dos eritrócitos totais (CTE) foram estabelecidos matematicamente os índices hematimétricos: volume corpuscular médio $\left(\mathrm{VCM}(\mathrm{fl})=\mathrm{HCT} \times 10 \div \mathrm{CTE} \times 10^{5}\right)$, hemoglobina corpuscular média (HCM (pg)= HGB x $10 \div$ CTE) e concentração de hemoglobina corpuscular média (CHCM $(\mathrm{g} / \mathrm{dL})=\mathrm{HGB} \mathrm{x}$ $100 \div$ HCT. O VCM e a CHCM foram determinados segundo (1996) e a HCM segundo Oliveira Lima et al. (1992).

A determinação do hematócrito (HCT) foi feita através da técnica do microhematócrito (Jain 2010). Para tanto, dois terços de um tubo capilar foi preenchido com a amostra e, após vedação com massa própria, centrifugado a $10.000 \mathrm{rpm}$ durante cinco minutos em uma centrífuga para microhematócrito (Eureka 1, BIO ENG ${ }^{\circledR}$ ). Com o auxílio de um cartão de hematócrito realizou-se a leitura do resultado em escala própria e expresso em porcentagem.

Análise estatística. Para avaliar as diferenças entre os resultados das amostras para as espécies estudadas, foi utilizado o teste t-Student $(\alpha=5 \%)$. A fim de estabelecer os limites de cada espécie para os parâmetros biométricos e hematológicos foi construído intervalos de confiança para as médias $(\alpha=5 \%)$. Uma análise de BiPlot com componentes principais foi utilizada para determinar quais os parâmetros avaliados, biométrico corporal e da cauda e hematológico, são responsáveis pela maior explicação da variância encontrada e assim poder diferenciar as espécies. Um gráfico de radar para os parâmetros biométricos e hematológicos também foi construído, para poder visualizar o perfil das espécies estudadas. As análises foram realizadas com o auxílio só software R.3.3.1.

\section{RESULTADOS}

Os 28 espécimes de Trachemys scripta elegans e 22 Trachemys dorbignyi estudadas eram adultas, hígidas e apresentavam, respectivamente, massa corporal média de $1498,02 \pm 446,60$ e $879,95 \pm 438,40$ gramas, respectivamente (Quadro 1), com intervalo de confiança de $(1348,41$; 1647,64 gramas) para T. scripta elegans e (705,53; 1054,37 gramas) para T. dorbignyi (Quadro 2) e comprimento máximo de carapaça médio, respectivamente, de $20,77 \pm 3,10 \mathrm{~cm}$ $(19,73 ; 21,82 \mathrm{~cm})$ e $18.79 \pm 2,50 \mathrm{~cm}(17,81 ; 19,77 \mathrm{~cm})$. Com exceção de $\mathrm{CpoC}$, as demais variáveis analisadas para os parâmetros biométricos apresentaram diferença significativa $(\mathrm{P}<0,05)$ entre as espécies (Quadro 1$)$.

Não houve diferença significativa $(\mathrm{P}>0,05)$ entre os valores médios obtidos no hemograma entre T. scripta elegans e T. dorbignyi criadas em cativeiro em Petrolina, PE (Quadro 1). 0 Quadro 2 exibe os intervalos de confiança com $95 \%$ de probabilidade para os valores do hemograma, considerando a distribuição $t$ de Student. 
Quadro 1. Valores de média + desvio-padrão para os parâmetros de biometria corporal e da cauda e do hemograma para tartarugas de orelhas vermelhas (T. scripta elegans) e tigres d'água (T. dorbignyi) criadas em cativeiro em Petrolina, PE, 2013

\begin{tabular}{ccc}
\hline Biometria corporal & T. scripta elegans & T. dorbignyi \\
\hline MC $(\mathrm{g})$ & $1498,02+446,60^{\mathrm{a}}$ & $879,95+438,40^{\mathrm{b}}$ \\
CMC $(\mathrm{cm})$ & $20,77+3,10^{\mathrm{a}}$ & $18,79+2,50^{\mathrm{b}}$ \\
LMC $(\mathrm{cm})$ & $17,08+1,60^{\mathrm{a}}$ & $14,64+1,80^{\mathrm{b}}$ \\
CMP $(\mathrm{cm})$ & $19,72+2,20^{\mathrm{a}}$ & $16,88+2,30^{\mathrm{b}}$ \\
LMP $(\mathrm{cm})$ & $13,30+1,30^{\mathrm{a}}$ & $11,32+1,50^{\mathrm{b}}$ \\
CTC $(\mathrm{cm})$ & $4,77+1,10^{\mathrm{b}}$ & $5,64+1,20^{\mathrm{a}}$ \\
CprC $(\mathrm{cm})$ & $2,02+0,80^{\mathrm{b}}$ & $3,14+0,70^{\mathrm{a}}$ \\
CpoC $(\mathrm{cm})$ & $2,76+0,60^{\mathrm{a}}$ & $2,70+0,40^{\mathrm{a}}$ \\
Hemograma & & \\
HCT $(\%)$ & $23,54+6,50^{\mathrm{a}}$ & $22,68+7,20^{\mathrm{a}}$ \\
HGB $(\mathrm{g} / \mathrm{dL})$ & $7,85+2,20^{\mathrm{a}}$ & $7,57+2,40^{\mathrm{a}}$ \\
CTE $\left(\mathrm{x} 10^{5} / \mu \mathrm{L}\right)$ & $6,49+2,60^{\mathrm{a}}$ & $5,73+2,40^{\mathrm{a}}$ \\
VCM $(\mathrm{fL})$ & $39,89+13,60^{\mathrm{a}}$ & $41,73+10,10^{\mathrm{a}}$ \\
CHCM $(\mathrm{pg})$ & $33,39+0,20^{\mathrm{a}}$ & $32,40+4,10^{\mathrm{a}}$ \\
HCM $(\%)$ & $13,30+4,50^{\mathrm{a}}$ & $14,88+5,50^{\mathrm{a}}$ \\
CGL $\left(\mathrm{x} 10^{3} / \mu \mathrm{L}\right)$ & $37,77+16,60^{\mathrm{a}}$ & $36,00+12,50^{\mathrm{a}}$ \\
\hline
\end{tabular}

Médias seguidas da mesma letra na mesma linha não diferem estatisticamente pelo teste $\mathrm{t}$ a $5 \%$ de probabilidade de erro. MC: massa corporal; CMC: comprimento máximo da carapaça; LMC: largura máxima da carapaça; CMP: comprimento máximo do plastrão; LMP: largura máxima do plastrão; CTC: comprimento total da cauda; CPrC: comprimento pré-cloacal; $\mathrm{CPoC}$ : comprimento pós-cloacal. HCT: hematócrito; HGB: Hemoglobina; CTE: Eritrócitos totais; VCM: Volume Corpuscular Médio; HCM: Hemoglobina Corpuscular Media; CHCM: Concentração de Hemoglobina Corpuscular Média; CGL: contagem global de leucócitos.

Quadro 2. Intervalos de confiança com $95 \%$ de probabilidade, considerando a distribuição t de Student para os parâmetros de biometria corporal e da cauda e de

hemograma para tartarugas de orelhas vermelhas (T. scripta elegans) e tigres d'água (T. dorbignyi) criadas em cativeiro em Petrolina, PE, 2013

\begin{tabular}{lcc}
\hline Biometria corporal & T. scripta elegans & T. dorbignyi \\
\hline MC $(\mathrm{g})$ & $(1348,41 ; 1647,64)$ & $(705,53 ; 1054,37)$ \\
CMC $(\mathrm{cm})$ & $(19,73 ; 21,82)$ & $(17,81 ; 19,77)$ \\
LMC $(\mathrm{cm})$ & $(16,55 ; 17,61)$ & $(13,92 ; 15,36)$ \\
CMP $(\mathrm{cm})$ & $(19,00 ; 20,44)$ & $(15,95 ; 17,81)$ \\
LMP $(\mathrm{cm})$ & $(12,87 ; 13,73)$ & $(10,73 ; 11,90)$ \\
CTC $(\mathrm{cm})$ & $(4,39 ; 5,15)$ & $(5,16 ; 6,13)$ \\
CprC $(\mathrm{cm})$ & $(1,76 ; 2,28)$ & $(2,85 ; 3,43)$ \\
CpoC $(\mathrm{cm})$ & $(2,55 ; 2,97)$ & $(2,54 ; 2,86)$ \\
Hemograma & & \\
HCT $(\%)$ & $(21,35 ; 25,73)$ & $(19,81 ; 25,56)$ \\
HGB $(\mathrm{g} / \mathrm{dL})$ & $(7,12 ; 8,58)$ & $(6,61 ; 8,53)$ \\
CTE $\left(\mathrm{x} 10^{5} / \mu \mathrm{L}\right)$ & $(5,61 ; 7,36)$ & $(4,79 ; 6,67)$ \\
VCM $(\mathrm{fL})$ & $(35,35 ; 44,43)$ & $(37,72 ; 45,73)$ \\
CHCM $(\mathrm{pg})$ & $(33,32 ; 33,45)$ & $(30,76 ; 34,05)$ \\
HCM $(\%)$ & $(11,79 ; 14,80)$ & $(12,69 ; 17,06)$ \\
CGL $\left(\mathrm{x} 10^{3} / \mu \mathrm{L}\right)$ & $(32,20 ; 43,34)$ & $(31,01 ; 40,99)$
\end{tabular}

Médias seguidas da mesma letra na mesma linha não diferem estatisticamente pelo teste $\mathrm{t}$ a $5 \%$ de probabilidade de erro. MC: massa corporal; CMC: comprimento máximo da carapaça; LMC: largura máxima da carapaça; CMP: comprimento máximo do plastrão; LMP: largura máxima do plastrão; CTC: comprimento total da cauda; $\mathrm{CPrC}$ : comprimento pré-cloacal; $\mathrm{CPoC}$ : comprimento pós-cloacal. HCT: hematócrito; HGB: Hemoglobina; CTE: Eritrócitos totais; VCM: Volume Corpuscular Médio; HCM: Hemoglobina Corpuscular Media; CHCM: Concentração de Hemoglobina Corpuscular Média; CGL: contagem global de leucócitos.

Correlação de Pearson significativa $(\mathrm{P}<0,05)$ foi observada entre os parâmetros biométricos corporais em $T$. scripta elegans e T. dorbignyi, sendo todos de forma posi- tiva (Quadro 3). Nos parâmetros relacionados à biometria caudal em T. scripta elegans foi observada correlação positiva significativa $(\mathrm{P}<0,05)$ entre o CTC e o $\mathrm{CprC}$ e CpoC, enquanto que em T. dorbignyi correlação positiva significativa $(\mathrm{P}<0,05)$ entre CprC e CTC; CprC e medidas da carapaça e plastrão e entre CpoC e MC (Quadro 3).

Em T. scripta elegans foi observada correlação de Pearson significativa $(\mathrm{P}<0,05)$ entre HCT e HGB, VCM, CHCM e HCM; entre HGB e VCM, CHCM e HCM; entre CTE e VCM e HCM e entre VCM e HCM e em T. dorbignyi entre HCT e HGB, CTE, CHCM; entre HB e CTE e CHCM; entre CTE e VCM e

Quadro 3. Correlação de Pearson entre os parâmetros biométricos corporais e da cauda em tartarugas de orelhas vermelhas (T. scripta elegans) e tigres d'água (T. dorbignyi) criadas em cativeiro em Petrolina, PE, 2013

\begin{tabular}{lccccccc}
\hline T. scripta elegans & CMC & LMC & CMP & LMP & CTC & CprC & CpoC \\
\hline CMC & - & - & - & - & - & - & - \\
LMC & $0,58^{* *}$ & - & - & - & - & - & - \\
CMP & $0,70^{* *}$ & $0,81^{* *}$ & - & - & - & - & - \\
LMP & $0,56^{* *}$ & $0,86^{* *}$ & $0,86^{* *}$ & - & - & - & - \\
CTC & 0,11 & $-0,12$ & 0,03 & $-0,06$ & - & - & - \\
CprC & 0,04 & $-0,26$ & $-0,13$ & $-0,31$ & $0,83^{* *}$ & - & - \\
CpoC & 0,04 & 0,11 & 0,13 & 0,27 & $0,61^{* *}$ & 0,09 & - \\
MC & $0,56^{* *}$ & $0,67^{* *}$ & $0,78^{* *}$ & $0,70^{* *}$ & 0,10 & $-0,02$ & 0,09 \\
T. dorbignyi & CMC & LMC & CMP & LMP & CTC & CprC & CpoC \\
CMC & - & - & - & - & - & - & - \\
LMC & $0,95^{* *}$ & - & - & - & - & - & - \\
CMP & $0,98^{* *}$ & $0,97^{* *}$ & - & - & - & - & - \\
LMP & $0,94^{* *}$ & $0,94^{* *}$ & $0,94^{* *}$ & - & - & - & - \\
CTC & 0,24 & 0,16 & 0,13 & 0,18 & - & - & - \\
CprC & $-0,25$ & $-0,27$ & $-0,30$ & $-0,33$ & $0,66^{* *}$ & - & - \\
CpoC & $0,53^{* *}$ & $0,54^{* *}$ & $0,52^{* *}$ & $0,55^{* *}$ & 0,43 & 0,11 & - \\
MC & $0,87^{* *}$ & $0,86^{* *}$ & $0,85^{* *}$ & $0,86^{* *}$ & 0,14 & $-0,34$ & $0,61^{* *}$
\end{tabular}

** significativo a $5 \%$ de probabilidade de erro pelo teste t. MC: massa corporal; CMC: comprimento máximo da carapaça; LMC: largura máxima da carapaça; CMP: comprimento máximo do plastrão; LMP: largura máxima do plastrão; CTC: comprimento total da cauda; $\mathrm{CprC}$ : comprimento pré-cloacal; CpoC: comprimento pós-cloacal.

Quadro 4. Correlação de Pearson entre os parâmetros

hematológicos em tartarugas de orelhas vermelhas (T. scripta elegans) e tigres d'água (T. dorbignyi) criadas em cativeiro em Petrolina, PE, 2013

\begin{tabular}{lcccccc}
\hline T. scripta elegans & HCT & HGB & CTE & VCM & CHCM & HCM \\
\hline HCT & - & - & - & - & - & - \\
HGB & $0,99^{* *}$ & - & - & - & - & - \\
CTE & 0,12 & 0,12 & - & - & - & - \\
VCM & $0,56^{* *}$ & $0,56^{* *}$ & $-0,68^{* *}$ & - & - & - \\
CHCM & $-0,45^{* *}$ & $-0,44^{* *}$ & $-0,06$ & $-0,30$ & - & - \\
HCM & $0,57^{* *}$ & $0,57^{* *}$ & $-0,66^{* *}$ & $1,00^{* *}$ & $-0,29$ & - \\
CGL & 0,24 & 0,24 & 0,26 & 0,02 & $-0,31$ & 0,02 \\
T. dorbignyi & HCT & HGB & CTE & VCM & CHCM & HCM \\
HCT & - & - & - & - & - & - \\
HGB & $0,99^{* *}$ & - & - & - & - & - \\
CTE & $0,56^{* *}$ & $0,56^{* *}$ & - & - & - & - \\
VCM & 0,31 & 0,31 & $-0,54^{* *}$ & - & - & - \\
CHCM & $0,52^{* *}$ & $0,53^{* *}$ & 0,44 & $-0,13$ & - & - \\
HCM & $-0,19$ & $-0,19$ & $-0,65^{* *}$ & $0,70^{* *}$ & $-0,80^{* *}$ & - \\
CGL & 0,19 & 0,19 & 0,29 & $-0,12$ & 0,01 & $-0,07$
\end{tabular}

** significativo a 5\% de probabilidade de erro pelo teste t. HCT: hematócrito; HGB: hemoglobina; CTE: contagem total de eritrócitos; VCM: volume corpuscular médio; CHCM: concentração de hemoglobina corpuscular média; HCM: hemoglobina corpuscular média; CGL: contagem global de leucócitos. 
Quadro 5. Correlação de Pearson entre os parâmetros biométricos corporais e da cauda e hematológicos em tartarugas de orelhas vermelhas (T. scripta elegans) e tigres d'água ( $T$. dorbignyi) criadas em cativeiro em Petrolina, PE, 2013

\begin{tabular}{lcccccccc}
\hline T. scripta elegans & MC & CMC & LMC & CMP & LMP & CTC & CPrc & CPoC \\
\hline HCT & 0.11 & $-0,10$ & $-0,14$ & $-0,16$ & $-0,18$ & 0,33 & 0,32 & 0,15 \\
HGB & 0.11 & $-0,09$ & $-0,14$ & $-0,16$ & $-0,18$ & 0,32 & 0,31 & 0,15 \\
CTE & -0.13 & $-0,03$ & $-0,22$ & $-0,21$ & $-0,34$ & $-0,10$ & 0,11 & $-0,31$ \\
VCM & 0.19 & $-0,05$ & 0,03 & 0,06 & 0,09 & 0,24 & 0,08 & 0,31 \\
CHCM & 0.15 & 0,22 & 0,10 & 0,24 & 0,13 & $-0,22$ & $-0,31$ & $-0,08$ \\
HCM & 0.57 & $-0,06$ & 0,02 & 0,05 & 0,08 & 0,24 & 0,08 & 0,29 \\
CGL & 0.24 & $-0,08$ & 0,00 & $-0,09$ & 0,04 & $-0,22$ & $-0,21$ & $-0,08$ \\
T. dorbignyi & MC & CMC & LMC & CMP & LMP & CTC & CPrc & CPoC \\
HCT & -0.19 & $-0,10$ & 0,00 & $-0,05$ & $-0,13$ & $-0,14$ & $-0,05$ & $-0,29$ \\
HGB & -0.19 & $-0,10$ & 0,00 & $-0,05$ & $-0,14$ & $-0,14$ & $-0,05$ & $-0,30$ \\
CTE & -0.26 & $-0,25$ & $-0,07$ & $-0,15$ & $-0,14$ & $-0,17$ & $-0,04$ & $-0,06$ \\
VCM & 0.19 & 0,34 & 0,21 & 0,26 & 0,19 & 0,20 & 0,06 & $-0,02$ \\
CHCM & -0.17 & $-0,14$ & $-0,07$ & $-0,03$ & $-0,13$ & $-0,29$ & $-0,18$ & $-0,43$ \\
HCM & 0.23 & 0,29 & 0,16 & 0,17 & 0,19 & 0,34 & 0,18 & 0,29 \\
CGL & -0.06 & $-0,26$ & $-0,26$ & $-0,28$ & $-0,22$ & 0,07 & 0,03 & $-0,06$
\end{tabular}

** significativo a $5 \%$ de probabilidade de erro pelo teste t. MC: massa corporal; CMC: comprimento máximo da carapaça; LMC: largura máxima da carapaça; CMP: comprimento máximo do plastrão; LMP: largura máxima do plastrão; CTC: comprimento total da cauda; CPrC: comprimento pré-cloacal; $\mathrm{CPoC}$ : comprimento pós-cloacal. HCT: hematócrito; HGB: Hemoglobina; CTE: Eritrócitos totais; VCM: Volume Corpuscular Médio; HCM: Hemoglobina Corpuscular Media; CHCM: Concentração de Hemoglobina Corpuscular Média; CGL: contagem global de leucócitos.

HCM; entre VCM e HCM e entre CHCM e HCM (Quadro 4). Não houve correlação significativa $(\mathrm{P}>0,05)$ entre os parâmetros biométricos corporais e da cauda e hematológicos em nenhuma das espécies estudadas (Quadro 5).

A análise de BiPlot indicou que a maior parte da variação encontrada entre as espécies pode é explicada por variáveis biométricas, sendo a LMP, CMP, LMC, CMC e MC, o que permitiu distinguir as espécies estudadas (Fig.1). A Figura 2 apresenta o perfil biométrico e sanguíneo das es-

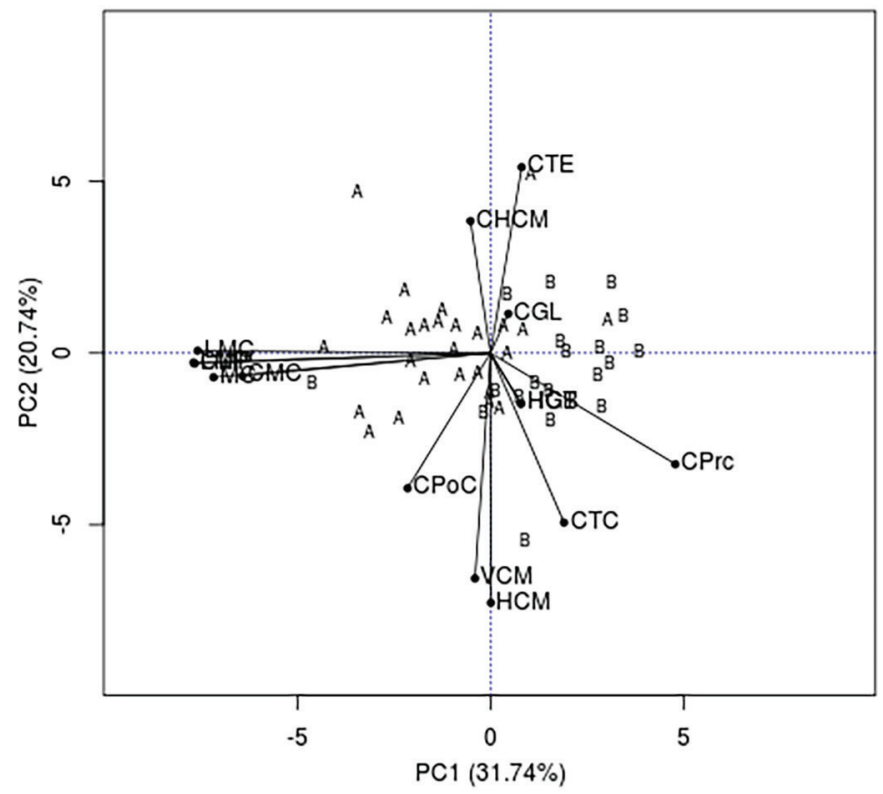

Fig.1. BiPlot para as variáveis biométricas corporais e da cauda e hematológicos para as espécies estudadas. (A) Trachemys scripta elegans e (B) Trachemys dorbignyi.
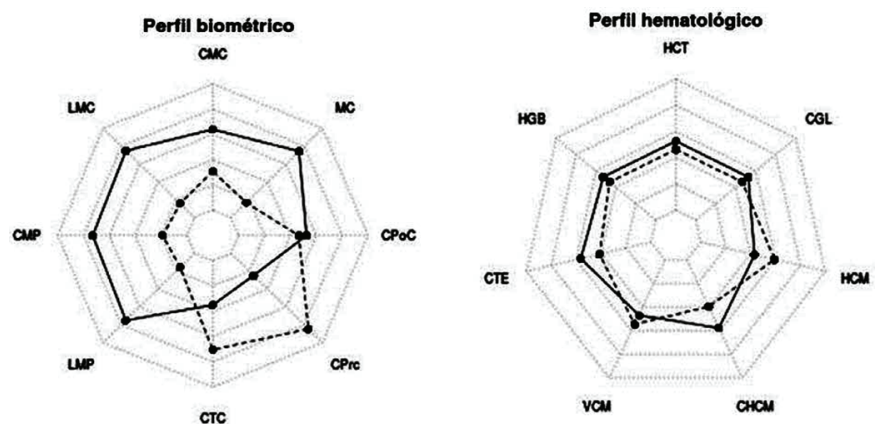

Fig.2. Starplots para o perfil biométrico e sanguíneo das espécies avaliadas. Linha contínua representa Trachemys scripta elegans e a linha tracejada indica Trachemys dorbignyi.

pécies estudadas e destaca a importância das variáveis que explicam a maior parte da variância encontrada entre as espécies como forma de distinguir as mesmas.

\section{DISCUSSÃO}

A despeito de estudos hematológicos comparativos de animais sadios e doentes fornecerem informações importantes para o manejo e conservação das espécies, sua aplicabilidade torna-se limitada quando não são estabelecidos padrões de normalidade da espécie. Isto ocorre porque muitos fatores podem levar a grandes variações nos testes realizados por diferentes laboratórios. Por isto este estudo avaliou os valores hematológicos em Trachemys scripta elegans e Trachemys dorbignyi criadas em condições de cativeiro no semiárido Nordestino brasileiro, buscando estabelecer parâmetros de referência para estas espécies nestas condições.

As tartarugas apresentavam carapaça, plastrão, cabeça, pescoço, cauda e membros livres de feridas e/ou aderências. 0 comprimento máximo da carapaça indicou que tanto T. scripta elegans (Rossi et al. 2006) quanto $T$. dorbinyi (Bager 2003) eram adultas. Os exemplares de $T$. scripta elegans possuíam massa corporal maior e CMC, LMC e CMP semelhante aos de fêmeas e superiores aos de machos e LMP ligeiramente superior aos de fêmeas e muito superior aos de machos desta espécie (Vieira \& Costa 2006). Já os T. dorbignyi estudados apresentaram tanto a massa corporal quanto CMC semelhante aos de machos desta espécie (Bager 2003), enquanto que o CTC e o CprC foram superiores aos de machos e fêmeas desta espécie (Silveira et al. 2012). Cabe ressaltar também que, embora os valores médios de massa corporal tenham sido inferiores aos de Silveira et al. (2012), os valores mínimo e máximo foram semelhantes. A alimentação regular e de qualidade, fornecida no cativeiro, parece ter sido a responsável pelos níveis de crescimento observados concordando com Gibbons (1967). A forte correlação positiva entre a MC e os parâmetros relacionados à carapaça e ao plastrão em ambas as espécies estudadas corroborou com Omonona et al. (2011) e indicou desenvolvimento harmônico entre massa corporal, carapaça e plastrão. Para T. scripta elegans as variáveis que mais contribuíram na diferenciação entre as espécies foram as variáveis biométricas MC, CMC, LMC, CMP e LMP, enquanto que em T. dorbignyi foram CTC e CprC. 
O hematócrito é um parâmetro importante nos exames de sangue, pois indica o percentual do sangue que é ocupado pelos eritrócitos circulantes em relação ao sangue total (McGavin \& Zachary 2011). Os valores médios observados em ambas as espécies encontraram-se entre os considerados normais em estudos com T. scripta elegans (Moon \& Foerster 2001, Jenkins-Perez 2012), indicando a ausência de hemoconcentração ou de anemia. Por outro lado, os valores deste estudo foram superiores aos obtidos através da veia coccígea dorsal por Medeiros et al. (2012), os quais observaram que a contaminação por fluido extracelular e pela linfa, que pode ocorrer quando a coleta de sangue é realizada através da veia coccígea dorsal, pode diminuir os valores de hematócrito, contagens total de eritrócitos e de leucócitos, assim como a concentração de hemoglobina, como também observado por Gottdenker \& Jacobson (1995). Os valores médios de hematócrito observados foram inferiores aos de tartarugas marinhas (Cubas \& Baptistotte 2006, Pires et al. 2009, Santos et al. 2009), provavelmente devido a uma adaptação fisiológica destas espécies ao ambiente (Moon \& Foerster 2001). Em relação a tartarugas de água doce, os valores foram semelhantes aos de Phrynops geofroanus (Zago et al. 2010, Silva 2011) e inferiores aos de Trachemys callirostris (Velásquez et al. 2014), ambos de áreas urbanas. A espécie, o ambiente e o manejo podem ter sido os responsáveis por estas diferenças (Stacy et al. 2011). Como um parâmetro derivado da medida da hemoglobina, o hematócrito reproduz seu valor na concentração sanguínea como evidenciado pela forte correlação positiva entre estes parâmetros tanto em T. scripta elegans $(\mathrm{r}=0,99)$ quanto em $T$. dorbignyi $(\mathrm{r}=0,99)$, corroborando com achados em peixes (Dias \& Sandrim 1998). Contudo, correlação entre hematócrito e contagem total de eritrócitos foi observada apenas em T. dorbignyi.

Os valores para a concentração média de hemoglobina em T. scripta elegans foram semelhantes aos de Medeiros et al. (2012) e Jenkins-Perez (2012). Os valores obtidos neste estudo foram semelhantes também aos de Chelonia mydas (Rossi et al. 2009, Santos et al. 2009) e Chelonia caretta (Cubas \& Baptistotte 2006, Pires et al. 2009); inferiores aos de Phrynops geoffroanus de áreas urbanas (Silva 2011) e ligeiramente superiores aos de T. callirostris (Velásquez et al. 2014). Brites (2002) observou influência do sexo e da área geográfica nas concentrações de hemoglobina, pois o maior tamanho das fêmeas seria o responsável pelas maiores concentrações de hemoglobina, fato que não pôde ser confirmado neste estudo, pois não houve distinção entre os sexos na realização dos exames. Correlação entre hemoglobina e contagem total de eritrócitos foi observada apenas em T. dorbignyi e entre hemoglobina e VCM e HCM apenas em T. scripta elegans. Outra diferença observada entre as espécies estudadas foi que a correlação entre hemoglobina e CHCM foi negativa em T. scripta elegans e positiva em $T$. dorbignyi.

A contagem total de eritrócitos não apresentou diferença significativa $(\mathrm{P}>0,05)$ entre T. scripta elegans e T. dorbignyi e esteve dentro da margem considerada normal para $T$. scripta elegans (Jenkins-Perez 2012, Medeiros et al. 2012) e Trachemys spp. (Alves 2013), onde os valores foram se- melhantes aos de P. geoffroanus (Zago et al. 2010) e T. callirostris (Velásquez et al. 2014) e superiores aos de $C$. caretta (Cubas \& Baptistotte 2006, Pires et al. 2009); C. mydas (Rossi et al. 2009, Santos et al. 2009) e P. geoffroanus (Silva 2011). Correlação negativa entre a contagem total de eritrócitos e VCM e HCM foi observada em ambas as espécies estudadas, todavia a ausência de correlação com a CHCM discordou de P. geoffroanus (Brites 2002).

Os valores de VCM, que representa o tamanho médio dos eritrócitos, foram muito inferiores aos obtidos em $T$. callirostris (Velásquez et al. 2014); C. caretta (Cubas \& Baptistotte 2006, Pires 2007); C. mydas (Santos et al. 2009) e P. geoffroanus (Brites 2002, Silva 2011). Este achado era esperado, pois estes autores observaram valores menores para a contagem de eritrócitos e maiores para o hematócrito (Cubas \& Baptistotte 2006, Santos et al. 2009, Pires et al. 2009, Silva 2011) e também porque a dieta, estado de saúde, exercícios, temperatura, estresse e hormônios circulantes podem afetar os resultados do VCM de tartarugas mantidas em cativeiro (Pires et al. 2006) e sugeriram que T. scripta elegans e T. dorbignyi possuem hemácias menores do que $C$. mydas, $C$. caretta, P.geoffroanus e T. callirostris. Em ambas as espécies foi observada correlação entre o VCM e o HCM .

Os valores de CHCM foram semelhantes aos de C. mydas (Santos et al. 2009); C. caretta (Cubas \& Baptistotte 2006) e $P$. geoffroanus (Zago et al. 2010) e superiores aos de $C$. caretta (Pires et al. 2009), P. geoffroanus (Silva 2011) e T. callirostris (Velásquez et al. 2014). Em mamíferos o CHCM geralmente acompanha o VCM (Garcia-Navarro \& Pachaly 1994), discordando deste estudo e de P. geoffroanus (Brites 2002).

Os valores de HCM foram muito superiores aos de $P$. geoffroanus (Zago et al. 2010) e extremamente inferiores aos de $C$ mydas (Santos et al. 2009); C caretta (Cubas \& Baptistotte 2006, Pires et al. 2009); P. geoffroanus (Brites 2002, Silva 2011) e T. callirostris (Velásquez et al. 2014). Acredita-se que as condições ambientais do semiárido nordestino brasileiro, assim como os valores de hemoglobina e de eritrócitos, que são utilizados no cálculo do HCM, influenciaram os resultados, como confirmado em T. scripta elegans que apresentaram os valores de HCM correlacionados positivamente com hemoglobina e negativamente com a contagem total de eritrócitos. Contudo em T. dorbignyi apenas a contagem total de eritrócitos foi negativamente correlacionada com o HCM. Apenas em T. dorbignyi houve correlação negativa entre HCM e CHCM.

A contagem global de leucócitos em T. scripta elegans e T. dorbignyi foi muito superior à observada em Trachemys spp. (Alves 2013); T. scripta elegans (Medeiros et al. 2012); C. mydas (Rossi et al. 2009, Santos et al. 2009); C. caretta (Cubas \& Batistotte 2006, Pires et al. 2009), P. geoffroanus (Brites 2002) e T. callirostris (Velásquez et al. 2014). Embora os resultados possam ser altamente variáveis em répteis, até mesmo quando se utiliza métodos consistentes de análise, a contagem global de leucócitos é um parâmetro muito útil quando se realizam estudos comparativos ou o monitoramento da evolução do quadro clínico (Work et al. 1998). A metodologia de contagem (Santos et al. 2009) e fatores 
como sexo (Brites 2002), estado nutricional ou estresse (Pires 2007, Santos et al. 2009); sazonalidade, distribuição geográfica (Duguy 1970, Santos et al. 2009); longa época de seca e altas temperaturas (Brites 2002) podem gerar valores diferentes. Acredita-se que todos estes fatores, com exceção do sexo, que não foi analisado neste estudo, poderiam explicar estas diferenças. Cabe ressaltar que Wood \& Ebanks (1984), não observaram em C. mydas diferenças entre os sexos na contagem global de leucócitos. Nenhum das espécies deste estudo foi observado correlação entre a contagem global de leucócitos e os outros parâmetros hematológicos.

\section{CONCLUSÕES}

Embora o estado nutricional, estresse, condições ambientais, dieta, estado de saúde, exercícios e temperatura possam influenciar os valores do hemograma, seu emprego é fundamental para obtenção de informações confiáveis e precisas sobre as condições de saúde em Testudines.

Os resultados demostram que a maior parte da variação observada entre Trachemys scripta elegans e Trachemys dorbignyi é explicada pelas variáveis biométricas e algumas correlações hematológicas caracterizam diferenças interespecíficas.

Conclui-se que os resultados lançam luz sobre valores de referência para estas espécies mantidas em cativeiro na região do semiárido e servem como um modelo para a fisiologia comparativa intra e interespécies.

Agradecimentos.- Os autores agradecem ao Centro de Triagem de Animais Silvestres (CETAS) do Parque Ecológico do Tietê, Guarulhos (SP), pela doação dos animais; e ao Laboratório de Análises Clínicas Veterinário ALPHA, Petrolina-PE, pela execução das análises.

\section{REFERÊNCIAS}

Almosny N.R.P. \& Monteiro A.O. 2007. Patologia clínica, p.939-966. In: Cubas Z.S., Silva J.C.R. \& Catão-Dias J.L. (Eds), Tratado de Animais Selvagens: medicina veterinária. Roca, São Paulo.

Alves G.M. 2013. Efeitos dos anticoagulantes - citrato de sódio, EDTA-K3 e heparina sódica nas análises hematimétricas e bioquímicas em araras Canindé (Ara ararauna), tigre d'água (Trachemys scripta) e Pacamãs (Lophiosilurus alexandri). Dissertação de Mestrado em Medicina Veterinária, Universidade Federal de Minas Gerais, Belo Horizonte, MG. 85p.

Bager A. 2003. Aspectos da biologia e ecologia da tartaruga tigre d'água, Trachemys dorbignyi (Testudines, Emydidae) no extremo sul do Rio Grande do Sul, Brasil. Tese de Doutorado em Ecologia, Universidade Federal do Rio Grande do Sul, Porto Alegre, RS. 100p.

Batistella A.M., Castro C.P. \& Vogt R.C. 2008. Geographic distribution, Trachemys adiutrix. Herpetol. Rev. 39:107-108.

Brites V.L.C. 2002. Hematologia, bioquímica do sangue, parasitologia, microbiologia, algas epizoárias e histopatologia de Phrynops geoffroanus (Schweigger, 1812) (Testudinata, Chelidae), expostos a diferentes influencias antrópicas no Rio Uberabinha, Minas Gerais. Tese de Doutorado em Ciências, Universidade Federal de São Carlos, São Carlos, SP. 196p.

Campbell T.W. 2004. Hematology of reptiles, p.259-276. In: Thrall M.A., Weiser G. \& Allison R.W. (Eds), Veterinary Hematology and Clinical Chemistry. Lippincott Williams and W.ilkins, Philadelphia.

Campbell T.W. 1996. ClinicaI pathology, p.248-257. In: Mader D.R. (Ed.), Reptile Medicine and Surgery. W.B. Saunders, London.

Casal A.B., Camacho M., L’Opez-Jurado L.F., Juste C. \& Orós J. 2009. Comparative study of hematologic and plasma biochemical variables in Eastern
Atlantic juvenile and adult nesting loggerhead sea turtles (Caretta caretta). Vet. Clin. Pathol. 38(2):213-218.

Cubas P.H. \& Baptistotte C. 2006. Chelonia (tartaruga, cágado e jabuti), p.86-119. In: Cubas Z.S., Silva J.C.R. \& Catão-Dias J.L. (Eds), Tratado de Animais Selvagens: medicina veterinária. Roca, São Paulo.

Deem S.L., Norton T.M., Mitchell M., Segars A., Alleman A.R., Cray C., Poppenga R.H., Dodd M. \& Karesh W.B. 2009. Comparison of blood values in foraging, nesting, and stranded loggerhead turtles (Caretta caretta) along the coast of Georgia, USA. J. Wildl. Dis. 45(1):41-56.

Dias M.T. \& Sandrim E.F.S. 1998. Características hematológicas de teleósteos brasileiros. I. Série vermelha e dosagens de cortisol e glicose do plasma sanguíneo de espécimes de Colossoma macropomum em condições de cultivo. Acta Scient. 20:157-160.

Divers S.J., Redmayne G. \& Aves K. 1996. Haematological and biochemical of 10 green iguanas (Iguana iguana). Vet. Rec. 138:203-205. (Apud Pires et al. 2009)

Duguy R. 1970. Number of blood cells and their variation, 93-109. In: Gans C. \& Parson T. (Eds), Biology of the Reptilian. Academic Press. New York. (Apud Brites 2002)

Falce M.C.L.B. 2000. Hematologia de répteis: revisão bibliográfica. Trabalho de Conclusão de Curso de Clínica Médica e Cirúrgica de Animais Selvagens e Exóticos, Instituto Quallitas, Campinas, SP. 53p.

Ferronato B.O. 2008. Phrynops geoffroanus (Testudines, Chelidae) em ambiente antrópico: perfil hematológico e microbiota oral. Dissertação de Mestrado em Ecologia Aplicada, Escola Superior de Agricultura Luiz de Queiroz, Piracicaba, SP. 64p.

Flint M., Morton J.M., Limpus C.J., Patterson-Kane J.C., Murray P.J. \& Mills P.S. 2010. Development and application of biochemical and haematolgical reference intervals to identify unhealthy green sea turtles (Chelonia mydas). Vet. J. 185(3):299-304.

Fonseca F.0. 2001. Olhares sobre o Lago Paranoá. Semarh, Brasília. 425p.

Fritz U. \& Hava P. 2007. Checklist of chelonians of the world. Vertebr. Zool. 57:149-368.

Garcia-Navarro C.E. \& Pachaly J.R. 1994. Manual de Hematologia Veterinária. Varela, São Paulo. 169p. (Apud Brites 2002)

Gibbons J.W., Scott D.E., Ryan T.J., Buhlmann .A., Tuberville T.D., Metts B.S., Greene J.L., Mills T., Leiden Y., Poppy S. \& Winne C.T. 2000. The global decline of reptiles, déjà vu amphibians. Bioscience 50(8):653-666.

Gibbons J.W. 1967. Variation in growth rates in three populations of the painted turtle, Chrysemys picta. Am. Midl. Nat. 79:517-519. (Apud Fagundes 2007)

Gottdenker N.L. \& Jacobson E.R. 1995. Effect of venipuncture sites on hematologic and clinical biochemical values in desert tortoises (Gopherus agassizii). Am. J. Vet. Res. 56: 19-21. (Apud Pires 2007)

Hawkey C.M. \& Dennett T.B. 1989. Hematology of reptiles, p.259-275. In: Ibid. (Eds), Comparative Veterinary Haematology. Wolfe Publishing Ltd, London. (Apud Falce 2009)

Hidalgo-Vila J., Díaz-Paniagua C., Pérez-Santigosa N., Plaza A., Camacho I. \& Recio F. 2007. Hematologic and biochemical reference intervals of free-living mediterranean pond turtles (Mauremys leprosa). J. Wildl. Dis. 43(4):798-801.

Jain N.C. 2010. Schalm's Veterinary Hematology. 6th ed. Wiley Blackwell, Iowa. $1206 \mathrm{p}$.

Jenkins-Perez J. 2012. Hematologic evaluation of reptiles: a diagnostic mainstay. Vet. Tech., August 2012:E1-E8.

McGavin M.D. \& Zachary J.F. 2011. Bases da patologia em veterinária. Elsevier, Brasil. 1496p.

Malvasio A., Gomes N. \& Farias E.C. 1999. Identificação sexual através do estudo anatômico do sistema urogenital em recém-eclodidos e jovens de Trachemys dorbignyi (Duméril et Bibron). Arq. Bras. Zootec. 16:91-102.

Medeiros N.C., Locatelli-Dittrich R., Schmidt E.M.S., Alvares A.A.A., Patricio L.L., Lange R.R. \& Souza R.A.M. 2012. Efeito do sítio de venopunção nos parâmetros hematológicos em tigre-d'água-americano, Trachemys scripta elegans. Pesq. Vet. Brasil. 32:37-40.

Metin K., Turozan O., Argin F., Basimoglu Koca Y., Taskavak E. \& Koca S. 
2006. Blood cell morphology and plasma biochemistry of the captive European Pond Turtle Emys orbicularis. Acta Vet. Brno 75:49-55.

Moon P.F. \& Foerster S.H. 2001. Zoological Restraint and Anesthesia: Reptiles, Aquatic Turtles (Chelonians). International Veterinary Information Service. Disponível em <http://pub.extranet.fsu.edu/sites/publicsafety/Wiki\%20Documents/Turtle\%20Handling.pdf> Acessado em 28 nov. 2015

Natt M.P. \& Herrick G.A. 1952. A new blood diluent for counting erythrocytes and leukocytes of the chicken. Poult. Sci. 31:735-738.

Oliveira Lima A., Soares J.B., Greco J.B., Galizzi J. \& Cançado J.R. 1992. Química do sangue, cap.2, p.2.1-2.61. In: Ibid. (Eds), Métodos de Laboratório Aplicado à Clínica: técnica e interpretação. Guanabara Koogan, Rio de Janeiro.

Omonona A.O., Olukole S.G. \& Fushe F.A. 2011. Haematology and serum biochemical parameters in free ranging African side neck turtle (Pelusios sinuatus) in Ibadan, Nigeria. Acta Herpetol. 6:267-274.

Owens D.W. \& Ruiz G.J. 1980. New methods of obtaining blood and cerebrospinal fluid from marine turtles. Herpetologica 36:17-20.

Padilla S.E., Weber M. \& Jacobson E. 2009. Comparación de anticoagulantes de heparina de litio y sodio en la bioquímica plasmática del cocodrilo de pantano (Crocodylus moreletii), en Campeche, México. Vet. México 40:203-2011.

Pires T.T. 2007. Avaliação do hemograma, bioquímica sérica e perfil eletroforético de proteínas de tartarugas marinhas Caretta caretta (Linnaeus, 1758) de vida livre e em cativeiro. Dissertação de Mestrado em Ciência Animal nos Trópicos, Universidade Federal da Bahia, Salvador, BA. 58p.

Pires T.T., Rostan G. \& Guimarães J.E. 2006. Hemograma e determinação da proteína plasmática total de tartarugas marinhas da espécie Caretta caretta (Linnaeus, 1758), criadas em cativeiro, Praia do Forte, Município de Mata de São João, Bahia. Braz. J. Vet. Res. Anim. Sci. 43:348-353.

Pires T.T., Rostan G., Bittencourt T.C.C. \& Guimarães J.E. 2009. Hemograma e bioquímica sérica de tartarugas cabeçudas (Caretta caretta) de vida livre e mantidas em cativeiro, no litoral norte da Bahia. Braz. J. Vet. Res. Anim. Sci. 46:11-18.

Primack R.B. \& Rodrigues E. 2001. Biologia da Conservação. Monograf, Londrina. 328p.

Rossi S., Lovato E. \& Höfling J.C. 2006. Aspectos biológicos da tartaruga-de-orelha-vermelha, Trachemys scripta elegans (Reptilia, Testudines, Emydidae) em cativeiro. Bioikos 20:33-40.

Rossi S., Sa-Rocha V.M., Kinoshita D., Genoy-Puerto A., Werneck M.R., Sa-Rocha L.C. \& Matushima E.R. 2009. Flow cytometry as a tool in the evaluation of blood leukocyte function in Chelonia mydas (Linnaeus, 1758) (Testudines, Cheloniidae). Braz. J. Biol. 69:899-905.
Ruiz G., Rosenmann M., Novoa F.F. \& Sabat P. 2002. Hematological parameters and stress index in rufous-collared sparrows dwelling in urban environments. Condor 104:162-166.

Santos M.R.D., Fereira L.S., Batistote C., Grossman A. \& Bellini C. 2009. Valores hematológicos de tartarugas marinhas Chelonia mydas (Linaeus, 1758) juvenis selvagens do Arquipélago de Fernando de Noronha, Pernambuco, Brasil. Braz. J. Vet. Res. Anim. Sci. 46:491-499.

Silva M.I.A. 2011. Alterações hematológicas e bioquímicas em uma população de Phrynops geoffroanus (Schweigger, 1812) em resposta a estressores ambientais. Dissertação de Mestrado em Genética, Instituto de Biociências, Letras e Ciências Exatas, Universidade Estadual Paulista, São José do Rio Preto, SP. 125p.

Silveira M.L., Hartmann M.T. \& Bager A. 2012. Biometria, razão sexual e dimorfismo sexual de Trachemys dorbigni (Duméril et Bibron, 1835) (Testudines, Emydidae) em um açude no município de São Gabriel, Rio Grande do Sul, Brasil. Biotemas 25(3):187-193.

Snoddy J.E., Landon M., Blanvillain G. \& Southwood A. 2009. Blood biochemistry of sea turtles captured in gillnets in the lower Cape Fear River, North Carolina, USA. J. Wildl. Manage. 73(8):1394-1401.

Stacy N.I., Alleman A.R. \& Sayler K.A. 2011. Diagnostic hematology of reptiles. Clin. Lab. Med. 31:87-108.

Tesserolli G.L. 2004. Análise hematológica e bioquimica em cágados pescoço-de-cobra (Hydromedusa tectifera) mantidos em cativeiro. Dissertação de Mestrado em Ciências Veterinárias, Universidade Federal do Paraná, Curitiba, PR. 69p.

Velásquez J.C., Cartagena H.N., Bolano C.R., Otero A.G., Pacheco J.C. \& Arias J.L. 2014. Caracterizacion hematologica de hicoteas (Trachemys callirostris Gray, 1856) en Cordoba, Colombia. Revta Med. Vet. 28:43-55.

Vieira C.S. \& Costa E.M.E. 2006. Análise da estrutura populacional de Trachemys scripta elegans (Chelonia) no Parque Ecológico Olhos D'água, Brasília, DF. Univ. Ciênc. Saúde 4:1-8.

Wood F.E. \& Ebanks G.K. 1984. Blood cytology and hematology of the green sea turtles, Chelonia mydas. Herpetologia 40(3):331-336. (Apud Brites 2002)

Work T.M., Raskin R.E., Balazs G.H. \& Whittaker S.D. 1998. Morphologic and cytochemical characteristics of blood cells from Hawaiian green turtles. Am. J. Vet. Res. 59:1252-1257. (Apud Pires 2007)

Zago C.E.S., Ferrarezi A.L., Vizotto L.D., Oliveira C., Cabral S.R.P., Taboga S.R., Bonilla-Rodriguez G.O., Venancio L.P.R. \& Bonini-Domingos C.R. 2010. Hemoglobin polymorphism and hematological profile of Geoffroy's sidenecked turtle (Phrynops geoffroanus, Testudines) in the northwestern region of São Paulo State, Brazil. Genet. Mol. Res. 9:721726. 